\title{
The binary nature of the Galactic centre X-ray source CXOGC J174536.1-285638
}

\author{
J. S. Clark ${ }^{1}$, P. A. Crowther ${ }^{2}$, and V. J. Mikles ${ }^{3}$ \\ 1 Department of Physics and Astronomy, The Open University, Walton Hall, Milton Keynes, MK7 6AA, UK \\ e-mail: jsc@star.ucl.ac.uk \\ 2 Department of Physics and Astronomy, University of Sheffield, Sheffield, S3 7RH, UK \\ 3 Department of Physics Astronomy, Louisiana State University, 273 Nicholson Hall, Tower Drive, Baton Rouge, LA 70803, USA
}

Received 30 June 2009 / Accepted 27 August 2009

\begin{abstract}
Context. The combination of X-ray and near-IR surveys of the central $2^{\circ} \times 0.8^{\circ}$ of the Galactic centre have revealed a population of X-ray bright massive stars. However, the nature of the X-ray emission, originating in wind collision zones or via accretion onto compact objects, is uncertain.

Aims. In order to address this we investigated the nature of one of the most luminous X-ray sources - CXOGC J174536.1-285638.

Methods. This was accomplished by an analysis of the near-IR spectrum with a non-LTE model atmosphere code to determine the physical parameters of the primary.

Results. This was found to be an highly luminous WN9h star, which is remarkably similar to the most massive stars found in the Arches cluster, for which comparison to evolutionary tracks suggest an age of 2-2.5 Myr and an initial mass of $\sim 110 M_{\odot}$. The X-ray properties of CXOGC J174536.1-285638 also resemble those of 3 of the 4 X-ray detected WN9h stars within the Arches and in turn other very massive WNLh colliding wind binaries, of which WR25 forms an almost identical "twin". Simple analytical arguments demonstrate consistency between the X-ray emission and a putative WN9h+mid O V-III binary, causing us to favour such a scenario over an accreting binary. However, we may not exclude a high mass X-ray binary interpretation, which, if correct, would provide a unique insight into the (post-SN) evolution of extremely massive stars. Irrespective of the nature of the secondary, CXOGC J174536.1-285638 adds to the growing list of known and candidate WNLh binaries. Of the subset of WNLh stars subject to a radial velocity survey, we find a lower limit to the binary fraction of $\sim 45 \%$; of interest for studies of massive stellar formation, given that they currently possess the highest dynamically determined masses of any type of star.
\end{abstract}

Key words. stars: early type - stars: binaries: general - Galaxy: center

\section{Introduction}

Near-IR observations of the Galactic centre have demonstrated that it hosts a large population of high mass stars, predominantly located within three young $(2-6 \mathrm{Myr})$, massive $\left(>10^{4} M_{\odot}\right)$ clusters in the central $\sim 50 \mathrm{pc}-$ the Arches, Quintuplet and Central cluster (e.g. Krabbe et al. 1995; Nagata et al. 1995; Cotera et al. 1996; Figer et al. 1996). Recently, the combination of radio, $\mathrm{X}$-ray and near-mid IR data has revealed an additional population of apparently isolated (candidate) massive stars throughout this region (e.g. Mauerhan et al. 2007). A full understanding of the processes governing star formation in the extreme environment of the Galactic centre therefore requires an explanation for their properties and origin - are they remnants of clusters disrupted by tidal forces or interaction with molecular clouds, the result of dynamical or $\mathrm{SNe}$ kick ejection from natal clusters or did they form in situ in a non clustered environment?

Identification of such stars has been facilitated by the production of deep X-ray catalogues of the Galactic centre (GC; Muno et al. 2006a, 2009), revealing over 9000 discrete point sources in the central $2^{\circ} \times 0.8^{\circ}$. Of these the majority are expected to be low mass systems, most likely cataclysmic variables, but a subset of the brighter, variable sources are expected to be either colliding wind binaries (CWBs) or high mass X-ray binaries (HMXBs). Cross correlation of these catalogues with near-IR imaging and subsequent spectroscopy has revealed a number of these sources to be identified with candidate high stellar mass counterparts (Muno et al. 2006b; Mauerhan et al. 2007). Critically, the star formation rate inferred for the GC suggest a statistically significant number of HMXBs (Muno et al. 2006a, and refs. therein) should be present and detectable, allowing the physical assumptions of population synthesis modeling (such as the SNe kick velocity) to be tested. Therefore, accurate classification of these systems is invaluable in order to confirm their nature and the origin of the X-ray emission.

Mikles et al. (2006, 2008, henceforth M06, M08) report the discovery of an emission line star associated with the bright X-ray source CXOGC J174536.1-285638 (abbreviated to CXO $\mathrm{J} 1745-28)$. The X-ray spectrum is dominated by strong Fe XXV emission, with the luminosity $\left(L_{\mathrm{x}} \sim 1.1 \times 10^{35} \mathrm{erg} \mathrm{s}^{-1}\right)$ and hard nature of the emission $\left(k T \sim 0.7_{-0.1}^{+0.1}+4.6_{-0.7}^{+0.7} \mathrm{keV}\right.$; M06) both arguing for a binary interpretation. However, the nature of the system (CWB or HMXB) remains obscure, even after the discovery of a $189 \pm 6 \mathrm{~d}$ periodicity in the X-ray flux (M08). This is largely due to the uncertainty regarding the properties of the primary and hence in this study we present a detailed analysis of the near-IR spectroscopy presented by M06 in order to provide accurate stellar parameters for the system primary and hence address 
the nature of the system. In Sect. 2 we present the results of this non-LTE analysis, discuss the nature of the system in Sect. 3 and summarise our results in Sect. 4.

\section{Observations and analysis}

We have carried out a non-LTE spectroscopic analysis of the near-IR spectroscopy of CXO J1745-28 obtained by M06 using IRTF/SpeX (Rayner et al. 2003) and CMFGEN (Hillier \& Miller 1998).

\subsection{IRTF/SpeX spectroscopy}

Short-wavelength, cross-dispersed spectroscopy of CXO J174528 was obtained by M06 on 1 Jul 2005 using IRTF/SpeX, covering the $J H K$ bands at a spectral resolution of $R \sim 1200$. M06 describe the data reduction process and spectral features which we shall not repeat here, except that the broad emission feature at $1.77 \mu \mathrm{m}$ attributed to He II (19-8) $1.772 \mu \mathrm{m}$ is in error. He II is expected to make a minor contribution to this feature, on the basis of negligible emission from He II (23-8) $1.658 \mu \mathrm{m}$ (218 is blended with He I $1.700 \mu \mathrm{m}$ ). The primary component is not known.

Crowther et al. (2006) discuss near-IR spectral classification of Wolf-Rayet stars from which we assign a WN9 subtype on the basis of $\mathrm{He} \mathrm{II} / \mathrm{Br} \gamma=0.06 \pm 0.01$. We may refine this subtype in view of its close morphological match to near-IR spectroscopy of HDE 313846 (WR108) from Bohannan \& Crowther (1999). HDE 313846 is a WN9ha star, for which the " $h$ " indicates the presence of significant hydrogen from the Pickering-Balmer decrement at visual wavelengths and the "a" represents intrinsic absorption lines in high Balmer lines. Analogous "h" diagnostics are available in the near-IR, such as the hydrogen Brackett series and corresponding He II $(n-8)$ transitions in CXO J1745-28. We therefore refine the subtype of CXO J1745-28 to WN9h. The morphological similarity between CXO J1745-28 and HDE 313846 suggests both are WN9ha subtypes, although no unambiguous diagnostic is available from low resolution near-IR spectroscopy. Other examples of WN9ha stars are known, such as HD 152408 (Bohannan \& Crowther 1999) which had earlier been classified as an Ofpe star by Walborn (1982), reflecting their proximity to the WolfRayet and Of boundaries. Recently, many other examples of WN7-9h stars have been identified in the Arches cluster from near-IR spectroscopy (Martins et al. 2008). Of these, F2 and F7 possess essentially identical $K$-band spectral morphologies to CXO J1745-28, while F6 only differs in its stronger C IV 2.070, $2.079 \mu \mathrm{m}$ features, as shown in Fig. 2. We also include one other WN9h star F9 for comparison since this is a strong X-ray source (as are F6 and F7), although its emission line spectrum is somewhat weaker.

We have used photometry of CXO J1745-28 from the fourth data release (DR4) of UKIDSS together with intrinsic $J H K$ colours predicted by our spectroscopic analysis (presented in Sect. 2.3), from which $K$-band extinctions, $A_{K}$, may be obtained using the extinction relations from Indebetouw et al. (2005). The UKIDSS project is defined in Lawrence et al (2007). UKIDSS uses the UKIRT Wide Field Camera (WFCAM; Casali et al, 2007). The photometric system is described in Hewett et al (2006), and the calibration is described in Hodgkin et al. (2009).

We favour UKIDSS photometry to 2MASS, owing to a latetype source which is blended with CXO J1745-28 in 2MASS datasets (see M08). Our derived extinction of $A_{K}=3.32 \mathrm{mag}$

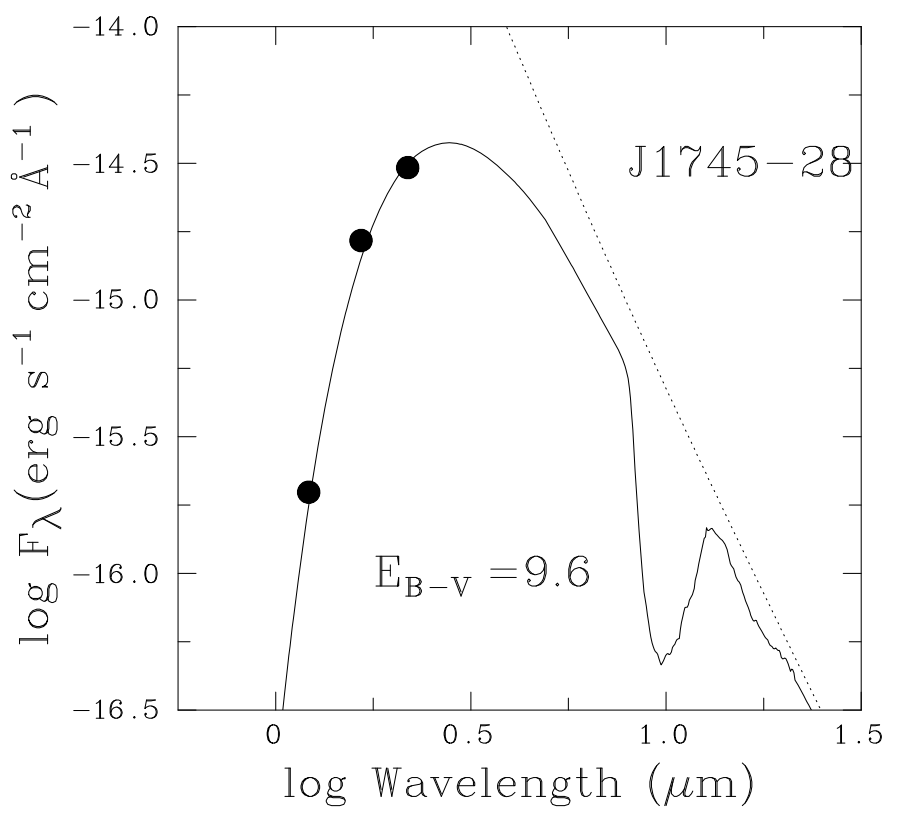

Fig. 1. Reddened model spectral energy distribution (SED) of CXO J1745-28 (solid line, $E_{B-V}=9.6$ ) and intrinsic SED (dotted line) overlaid upon IR photometry from UKIDSS.

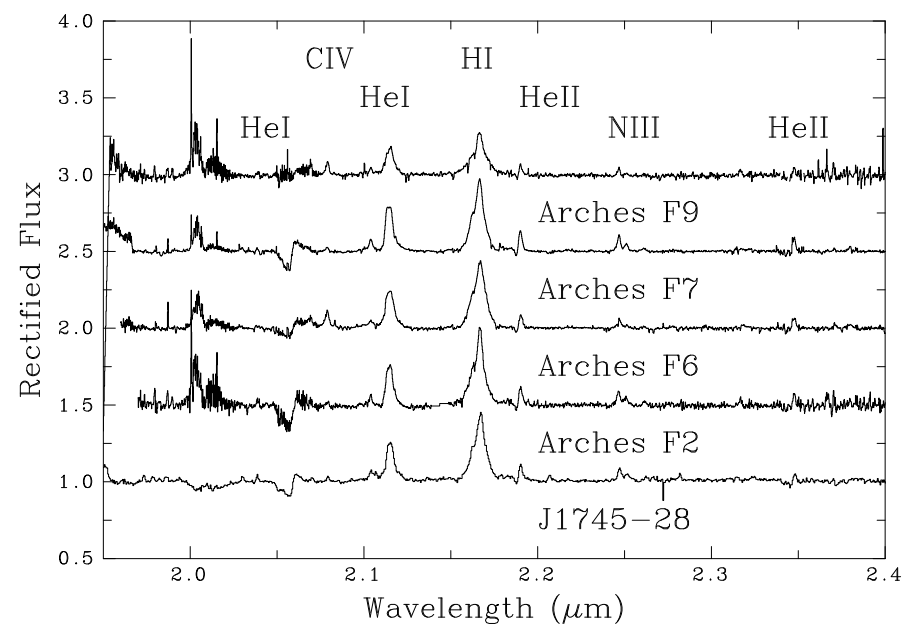

Fig. 2. $K$-band spectroscopy of CXO J1745-28 from M06 together with morphologically similar WN9h stars from the Arches cluster (F2, F6 and F7) plus the weaker lined WN9h star F9 which is X-ray bright, as are F6 and F7. The VLT/SINFONI Arches spectroscopy is from Martins et al. (2008).

for CXO J1745-28 corresponds to $E_{B-V}=9.6$ or $A_{V}=30 \mathrm{mag}$, assuming a standard Galactic extinction law. For an assumed distance of $8 \mathrm{kpc}$ to the Galactic Centre (Reid 1993), we obtain an absolute $K$-band magnitude of -7.51 for CXO J174528. In Fig. 1 we present the spectral energy distribution (SED) of our J1745-28 model, reddened according to the IR extinction law presented in Morris et al. (2000), together with UKIDSS photometry. Spitzer IRAC photometry is not available, although the theoretical model suggests $[3.6]=8.28,[4.5]=7.66$, $[5.8]=7.24$ and $[8]=7.11$ for J1745-28. Our derived extinction agrees well with M06 who estimated $A_{V}=29$ mag by simply adopting $(H-K)_{0}=0.0$ mag.

For comparison, WN9h stars within the Arches cluster possess HST/NICMOS F205W magnitudes in the range 10.45 (F1) to 11.2 (F2) based upon photometry from Figer et al. (2002). 
Table 1. UKIDSS near-IR photometry, extinction and absolute magnitude determinations for an assumed distance of $8 \mathrm{kpc}$ to CXO J1745-28 (Reid 1993).

\begin{tabular}{lllllllllll}
\hline \hline Star & $K$ & $J-K$ & $H-K$ & $(J-K)_{0}$ & $(H-K)_{0}$ & $A_{K}^{J-K}$ & $A_{K}^{H-K}$ & $A_{K}$ & DM & $M_{K}$ \\
\hline CXO J1745-28 & 10.40 & 5.07 & 1.78 & -0.04 & 0.01 & 3.42 & 3.22 & 3.32 & 14.52 & -7.51 \\
\hline
\end{tabular}

These correspond to absolute $F 205 W$ magnitudes of -6.4 to $-7.2 \mathrm{mag}$ for a uniform $K$-band extinction of $A_{\mathrm{K}_{\mathrm{s}}}=3.1 \mathrm{mag}$ (Kim et al. 2006) and (identical) distance of $8 \mathrm{kpc}$. As such, one would expect that CXO J1745-28 to be amongst the most luminous of the WN9h stars in the Galactic Centre region.

\subsection{Atmospheric code}

The non-LTE atmosphere code CMFGEN solves the radiative transfer equation in the co-moving frame, under the additional constraints of statistical and radiative equilibrium.

Since CMFGEN does not solve the momentum equation, a density or velocity structure is required. For the supersonic part, the velocity is parameterized with a classic $\beta$-type law. This is connected to a hydrostatic density structure at depth, such that the velocity and velocity gradient match at the interface. The subsonic velocity structure is defined by a corresponding $\log g=$ 3.25 fully line-blanketed plane-parallel TLUSTY model (Lanz \& Hubeny 2003).

CMFGEN incorporates line blanketing through a super-level approximation, in which atomic levels of similar energies are grouped into a single super-level which is used to compute the atmospheric structure. Our atomic model is similar to that adopted by Crowther et al. (2002), including ions from H I, He III, C III-IV, N III-V, O III-VI, Si IV, P IV-V, S IV-VI and Fe IV-VII. By number, the main contributors to line blanketing are Fe IV-V. In addition, extended model atoms of $\mathrm{C}$ III, N III and $\mathrm{O}$ III were included since each contribute to the $2.112 \mu \mathrm{m}$ emission feature.

We have assumed a depth-independent Doppler profile for all lines when solving for the atmospheric structure in the comoving frame, while in the final calculation of the emergent spectrum in the observer's frame, we have adopted a uniform turbulence of $50 \mathrm{~km} \mathrm{~s}^{-1}$. Incoherent electron scattering and Stark broadening for hydrogen and helium lines are adopted.

With regard to wind clumping, this is incorporated using a radial dependent volume filling factor, $f$, as described in Hillier et al. (2003), with a typical value of $f=0.1$ resulting in a reduction in mass-loss rate by a factor of $\sqrt{(1 / f)} \sim 3$.

\subsection{Analysis}

We derive the stellar temperature CXO J1745-28 using diagnostic He I $2.058 \mu \mathrm{m}, 1.700 \mu \mathrm{m}$, He II $2.189 \mu \mathrm{m}, 1.692 \mu \mathrm{m}$ together with $\mathrm{Br} \gamma$ for the mass-loss rate, hydrogen content and velocity structure. The P Cygni He II $2.189 \mu$ m profile naturally arises in the stellar wind of the star and does not require the presence of an additional source of ionisation within the system, such as a compact companion (e.g. M06). Stellar temperatures, $T_{*}$, correspond to a Rosseland optical depth of 20 , which are typically (up to) a few thousand degrees higher than effective temperatures, $T_{2 / 3}$, relating to optical depths of $2 / 3$ in such stars.

We have estimated a terminal wind velocity of $1350 \mathrm{~km} \mathrm{~s}^{-1}$ from He I $2.058 \mu \mathrm{m}$ from which a (slow) velocity law of exponent $\beta=1.5$ is used for the supersonic velocity structure. $\beta=0.8$ was adopted by Martins et al. (2008), for which similar synthetic spectra are predicted, except for somewhat weaker
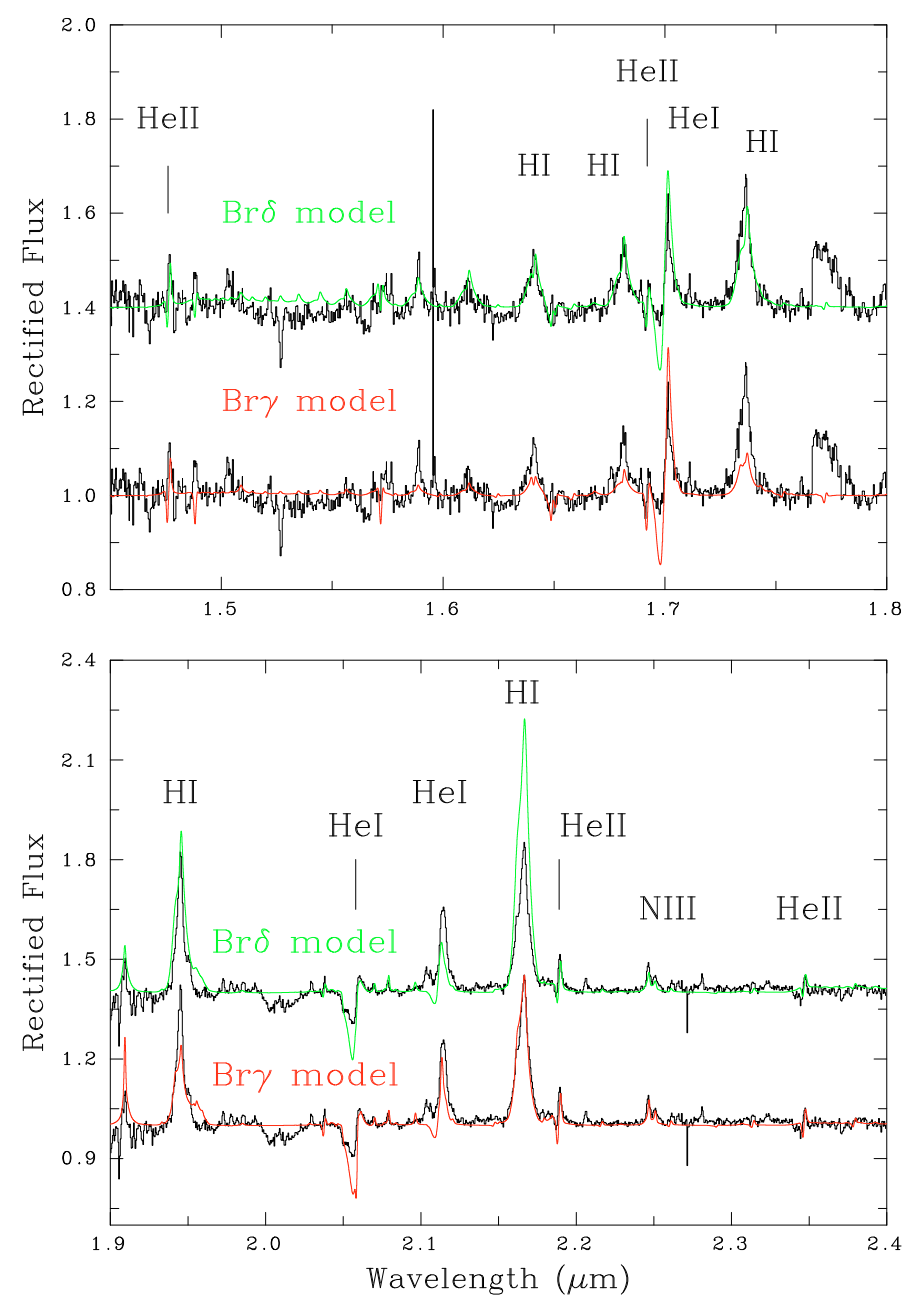

Fig. 3. $H$ and $K$ band IRTF/SpeX observations of CXO J1745-28 from M06 (black) with our $\mathrm{Br} \gamma$-derived (red, $X_{\mathrm{H}}=25 \%$ ) and $\mathrm{Br} \delta$-derived (green, $X_{\mathrm{H}}=50 \%$ ) synthetic spectra overplotted.

emission and absorption lines. A measure of the nitrogen mass fraction is obtained from the weak N III $2.247,2.251 \mu \mathrm{m}$ doublet since the stronger feature at $2.112 \mu \mathrm{m}$ is blended with He I, C III and O III, while C IV 2.070, $2.079 \mu \mathrm{m}$ allows an estimate of the carbon content. We adopt solar values for all other metals (e.g. Cox 2000; with the exception of oxygen for which the value given by Asplund et al. 2004, was used). Spectroscopic fits to IRTF/SpeX observations are presented in Fig. 3.

Overall the agreement between predicted line profiles and observations is satisfactory, although He I $2.058 \mu \mathrm{m}$ and $1.700 \mu \mathrm{m}$ P Cygni absorption are too strong in the synthetic $\operatorname{spectrum}(\beta=0.8$ does provide an improved match to $2.058 \mu \mathrm{m})$ and it is apparent that the high members of the hydrogen Brackett series are predicted to be too weak in emission for the adopted $X_{\mathrm{H}}=25 \%$ by mass $(\mathrm{He} / \mathrm{H}=0.7$ by number $)$. This model is referred to as the $\mathrm{Br} \gamma$ model in Table 2, although it should be emphasised that He I lines within the 7-4 set of transitions contribute $\sim 30 \%$ of the equivalent width for this feature. 
Table 2. Derived physical and wind parameters for CXO J1745-28 (WN9h) for CWB and HMXB scenarios.

\begin{tabular}{llllllllllll}
\hline \hline Scenario & Model & $\begin{array}{l}T_{*} \\
\mathrm{kK}\end{array}$ & $\begin{array}{l}R_{*} \\
R_{\odot}\end{array}$ & $\begin{array}{l}T_{\text {eff }} \\
\mathrm{kK}\end{array}$ & $\begin{array}{llll}\log L_{*} \\
L_{\odot}\end{array}$ & $\begin{array}{l}v_{\infty} \\
\mathrm{km} \mathrm{s}^{-1}\end{array}$ & $\begin{array}{l}\log \mathrm{d} M / \mathrm{d} t \\
M_{\odot} \mathrm{yr}^{-1}\end{array}$ & $\begin{array}{l}M_{K_{\mathrm{s}}} \\
\mathrm{mag}\end{array}$ & $\begin{array}{l}X_{\mathrm{H}} \\
\%\end{array}$ & $\begin{array}{l}X_{\mathrm{He}} \\
\%\end{array}$ & $\begin{array}{l}X_{\mathrm{N}} \\
\%\end{array}$ \\
\hline CWB & $\mathrm{Br} \gamma$ & 32.0 & 43.1 & 30.9 & 6.24 & 1350 & -4.46 & -7.42 & 25 & 74 & 1.2 \\
& $\mathrm{Br} \delta$ & 32.5 & 41.8 & 30.5 & 6.24 & 1350 & -4.40 & -7.42 & 50 & 49 & 1.2 \\
HMXB & $\mathrm{Br} \gamma$ & 32.0 & 44.9 & 30.9 & 6.28 & 1350 & -4.43 & -7.51 & 25 & 74 & 1.2 \\
& $\mathrm{Br} \delta$ & 32.5 & 43.5 & 30.5 & 6.28 & 1350 & -4.37 & -7.51 & 50 & 49 & 1.2 \\
\hline
\end{tabular}

In order to better reproduce the strength of the higher Brackett series, a significantly higher hydrogen content of $X_{\mathrm{H}}=50 \%$ by mass $(\mathrm{He} / \mathrm{H}=0.25$ by number $)$ is required, which is also presented in Fig. 3. Model parameters for this, our $\operatorname{Br} \delta$ model, are also presented in Table 2. Consequently, one should caution against the use of a solitary diagnostic in view of the significant difference resulting from other diagnostics even for cases as simple as a single hydrogen series.

In both cases, we estimate a nitrogen mass fraction of $1.2 \%$ for CXO J1745-28 from the N III 2.247, $2.251 \mu \mathrm{m}$ features which should be reliable to $\pm 50 \%$. Carbon is poorly constrained, although $X_{\mathrm{C}}=0.03 \%$ by mass reproduces the weak C IV 2.070, $2.079 \mu \mathrm{m}$ features satisfactorily.

In view of the uncertain nature of the companion to the WN9h star (Sect. 3), we have estimated its stellar properties under two assumptions; either the near-IR spectrum of the WolfRayet star is (weakly) diluted by the continuum of a massive companion in the case of a CWB system or it suffers negligible contribution from the compact companion in the case of a HMXB. Here, we adopt a mass ratio $q=0.4$, which is consistent with $q \geq 0.2$ favoured by M08 for a sole dominant IR source. For a current WN9h mass of $80 M_{\odot}$ (see below), we shall use $30 M_{\odot}$ for the companion mass, namely an O6 dwarf, O7 giant or O9 supergiant from the Martins et al. (2005) O star calibration. On the basis of the companion wind properties, discussed in Sect. 3, we shall adopt O7 III for the putative companion, for which $M_{K}=-4.76$ (Martins \& Plez 2006). For a systemic absolute magnitude of $M_{K}=-7.51 \mathrm{mag}$ we obtain $M_{K}=-7.42$, i.e. the light ratio of the $\mathrm{O}$ to WR star is 0.09 in the $K$-band $(0.10$ in the $J$ and $H$ bands). Derived stellar parameters for the WN9h star in these scenarios are presented in Table 2.

\subsection{Comparison with Arches cluster members}

The position of CXO J1745-28 on the H-R diagram is presented in Fig. 4 together with $\mathrm{O}$ supergiant and WN7-9h members of the Arches cluster from Martins et al. (2008) and solar metallicity, rotating $\left(300 \mathrm{~km} \mathrm{~s}^{-1}\right)$ theoretical models from Meynet \& Maeder (2000). Note that the stellar luminosities of the Arches members have been downward corrected by 0.1 dex due to an error in the original study (Martins, priv. comm.), but re-adjusted upward by 0.05 dex for consistency with our adopted distance ${ }^{1}$. Arches WN9h members F2, F6 and F7 are of particular interest in view of their spectroscopic similarities to CXO J1745-28 (recall Fig. 2), for which Martins et al. (2008) obtained stellar temperatures of $34 \mathrm{kK}$ (versus $32 \mathrm{kK}$ here for CXO J1745-28). However, $K$-band bolometric corrections, $\mathrm{BC}_{K}$, for the Arches members remain significantly larger $\left(\mathrm{BC}_{\mathrm{F} 205 \mathrm{~W}} \sim-4 \mathrm{mag}\right)$ than our estimate for $\mathrm{J} 1745-28\left(\mathrm{BC}_{K} \sim-3.5 \mathrm{mag}\right)$, even after the 0.1 dex correction in stellar luminosity. If we turn to abundance

\footnotetext{
${ }^{1}$ Martins et al. (2008) adopted a distance of $7.6 \mathrm{kpc}$ to the Arches cluster, a $K$-band extinction of 2.8 mag and F205W photometry from Figer et al. (2002).
}

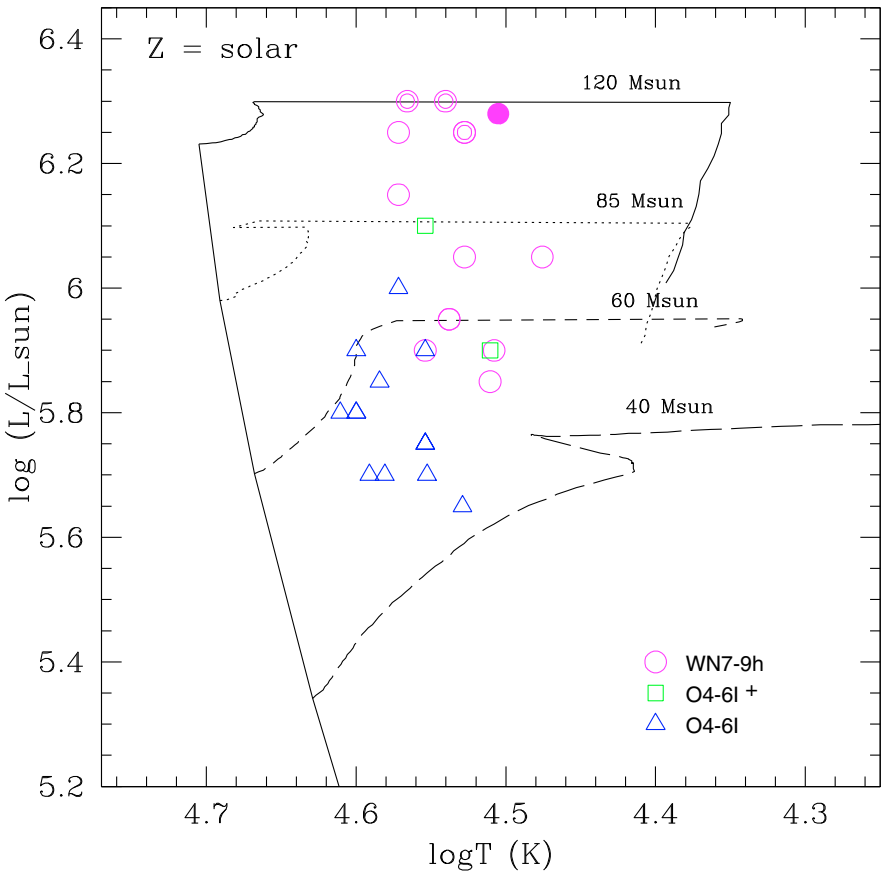

Fig. 4. HR diagram indicating the position of CXO J1745-28 (filled symbol) and the WN7-9ha and O4-6I members of the Arches cluster (open symbols; Martins et al. 2008), with the luminosity of Arches members revised downwards by $0.05 \mathrm{dex}$ as described in Sect. 2.4. The Geneva evolutionary tracks for rotating $\left(V_{\text {rot }}=300 \mathrm{~km} \mathrm{~s}^{-1}\right) 40-120 M_{\odot}$ stars at solar metallicity are overplotted (Meynet \& Maeder 2000). The Arches stars F6, $7 \& 9$ (CWBs based on their X-ray properties, see Sect. 3) are indicated by the double rings.

estimates, $\mathrm{Br} \gamma$-derived surface hydrogen contents in the range $40 \% \leq X_{\mathrm{H}} \leq 55 \%$ are obtained for $\mathrm{F} 2, \mathrm{~F} 6$ and $\mathrm{F} 7$, versus $25 \%$ for CXO J1745-28, while the nitrogen mass fraction obtained for CXO J1745-28 is also at the lower end of the range observed for the Arches members; $1.2 \%$ versus $1.1-2.8 \%$ respectively.

We infer an initial (current) mass of $\sim 110(80) M_{\odot}$ and a current age of 2-2.5 Myr for CXO J1745-28, such that it is likely a very massive star that is slowly evolving away from the hydrogen burning main sequence. As such, J1745-28 is not a classical helium-burning Wolf-Rayet star.

Predicted surface hydrogen mass fractions at this early stage are $X_{\mathrm{H}}=40 \%$ for models initially rotating at $300 \mathrm{~km} \mathrm{~s}^{-1}$, with lower depletions of $X_{\mathrm{H}}=65-70 \%$ for equivalent non-rotating models. In Fig. 5 we compare the $\mathrm{Br} \gamma$-derived surface hydrogen content for CXO J1745-28 with Arches cluster members from Martins et al. (2008) and the predicted surface hydrogen from Meynet \& Maeder (2000) models. Recall that the hydrogen content for CXO J1745-28 depends upon the diagnostic hydrogen line used, either $X_{\mathrm{H}}=25 \%$ from $\mathrm{Br} \gamma$ (the same diagnostic as used by Martins et al. 2008) or $X_{\mathrm{H}}=50 \%$ for higher Brackett lines. 
Table 3. Summary of the properties of CXO J17545-28 in comparison to magnetic O and WR and Of?p stars.

\begin{tabular}{|c|c|c|c|c|c|}
\hline$\overline{\mathrm{ID}}$ & $\begin{array}{l}\text { Spec. } \\
\text { type }\end{array}$ & $\begin{array}{l}\log \left(L_{\mathrm{bol}}\right) \\
\left(\mathrm{erg} \mathrm{s}^{-1}\right)\end{array}$ & $\begin{array}{l}\log \left(L_{\mathrm{X}}\right) \\
\left(\operatorname{erg~s}^{-1}\right)\end{array}$ & 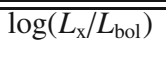 & $\overline{\text { Ref. }}$ \\
\hline CXO J1745-28 & WN9h & 39.8 & 35.0 & -4.8 & 1 , this study \\
\hline$\theta^{1}$ Ori C & $\mathrm{O} 7 \mathrm{~V}$ & 39.0 & 33.0 & -6.0 & $2,3,4$ \\
\hline$\zeta$ Ori A & O9.7Ib & 38.8 & 32.1 & -6.7 & 5,6 \\
\hline HD 108 & $\mathrm{O} 4 \mathrm{f} ? \mathrm{p} \leftrightarrow 08.5 \mathrm{fp}$ & 39.2 & 33.1 & -6.1 & 2,3 \\
\hline HD 148937 & O5.5-6f?p & 39.3 & 33.3 & -6.0 & 3,7 \\
\hline HD $152408(=W R$ 79a) & WN9ha & 39.6 & 31.9 & -7.7 & 8 \\
\hline HD 164794 & O4 V((f)) & 39.5 & 33.1 & -6.4 & 9 \\
\hline HD 191612 & O6.5f?pe $\leftrightarrow$ O8fp & 39.1 & 32.9 & -6.1 & 2 \\
\hline
\end{tabular}

Note that no magnetic field determinations have been made for HD 108, while HD 164974 (=9 Sgr) is a suspected CWB. References for this table are: (1) Mikles et al. (2006); (2) Náze et al. (2007); (3) Náze et al. (2008b); (4) Gagné et al. (2005); (5) Raassen et al. (2008); (6) Bouret et al. (2008); (7) Náze et al. (2008c); (8) Oskinova (2005); and (9) Rauw et al. (2002).

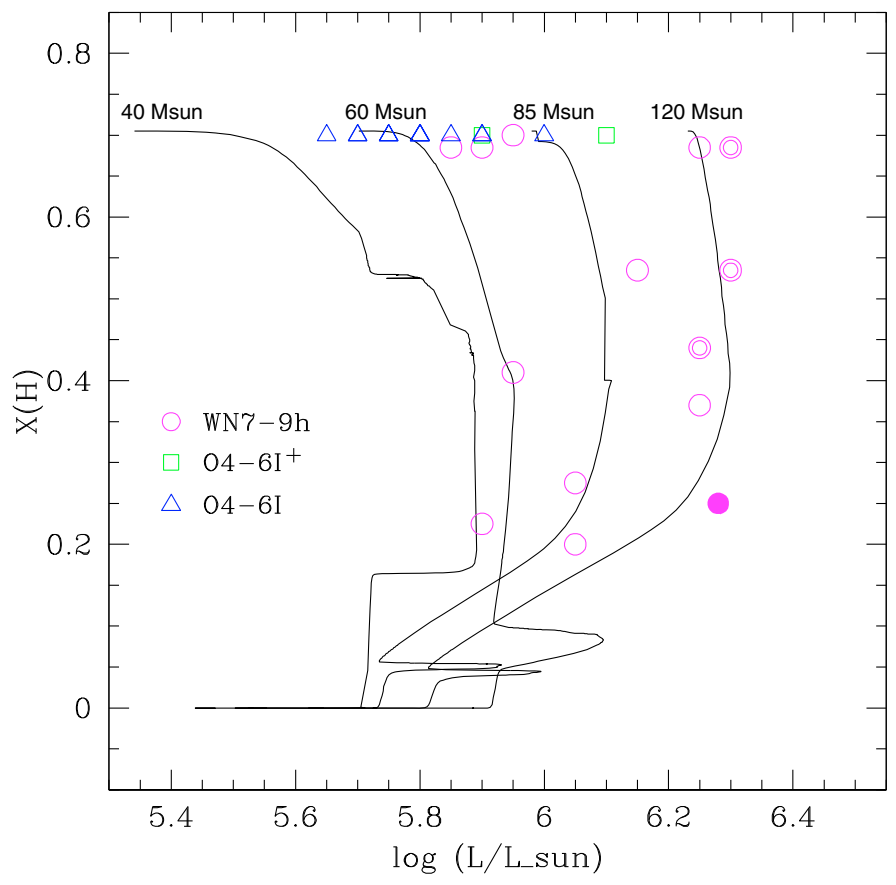

Fig. 5. Comparison of the Hydrogen mass fraction as a function of luminosity for CXO 1745-28 and the stellar population of the Arches cluster, with symbols having the same meaning as in Fig. 4. To enable a direct comparison to the Arches population the results from the $\mathrm{Br} \gamma$ model are presented $-X_{\mathrm{H}}=50 \%$ by mass for the $\operatorname{Br} \delta$ model (Sect. 2.3). Again, Geneva evolutionary tracks for rotating $\left(v_{\text {rot }}=300 \mathrm{~km} \mathrm{~s}^{-1}\right)$ 40-120 $M_{\odot}$ stars at solar metallicity have been overplotted (Meynet \& Maeder 2000).

\section{The nature of CXO J1745-28 - CWB or HMXB?}

Our classification of the primary in CXO J1745-28 as a WN9h star permits a more accurate comparison to the properties of both single stars and known HMXBs and CWBs. The ratio of X-ray to bolometric luminosity of CXO J1745-28 - $\log \left(L_{\mathrm{X}} / L_{\mathrm{bol}}\right) \sim-4.8$ - is significantly in excess of that observed for both single $\mathrm{O}$ and WR stars $-\log \left(L_{\mathrm{x}} / L_{\mathrm{bol}}\right) \sim-7-$ where the emission is thought to arise in shocks embedded in the stellar wind. Recent work has shown that a number of $\mathrm{O}$ stars have measureable magnetic fields (Donati et al. 2002, 2006; Hubrig et al. 2008; Bouret et al. 2008), thus raising the possibility that the magnetically confined wind shock model of Babel \& Montmerle et al. (1997a,b) might be applicable and lead to the production of significant X-ray emission. In Table 3 we summarise the X-ray properties of these stars as well as the Of?p star HD 108 (motivated by the fact that the other three examples of Of?p stars all have detectable magnetic fields).

While these stars appear to show excess X-ray emission with respect to field $\mathrm{O}$ and WR stars (Table 3; Náze et al. 2007), the ratio of X-ray to bolometric luminosity is over an order of magnitude lower than CXO J1745-28, with the WN9ha star HD 152408 (=WR 79a) being significantly fainter still. Moreover, while the X-ray spectra of the magnetically active stars are also consistent with a multitemperature model, both cool and hot components are systematically cooler than found for CXO J1745-28 (Rauw et al. 2002; Gágne et al. 2005; Naze et al. 2004, 2007, 2008c). Finally, these stars demonstrate spectral variability attributed to an asymmetric circumstellar environment caused by the entrainment of the stellar wind by the magnetic field (e.g. Náze et al. 2008b). However, spectroscopy of CXO J1745-28 indicates there is no evidence for a departure from spherical symmetry, while no variability has currently been observed (Mikles et al. 2006, 2008). Therefore, we conclude that the X-ray emission in CXO J1745-28 most likely arises as a result of binarity.

Assuming the $189 \mathrm{~d}$ periodicity is orbital and a canonical accretion efficiency $\epsilon \sim 0.1$, the wind properties imply a compact companion mass of $\sim 5 M_{\odot}$ for a HMXB interpretation (Eq. (10); M08). Three HMXBs with moderately evolved very massive companions are known; 4U1700-37, OAO1657-415 \& GX301-2 ${ }^{2}$. All are more X-ray luminous than CX J1745-28; as expected, given their smaller orbital separations. However their X-ray spectra - a power law with high energy cutoff, with no 6.7 keV Fe XXV line (White et al. 1983; La Barbera et al. 2005; Audley et al. 2006) - are significantly different ${ }^{3}$.

Motivated by the similarity in X-ray properties of $\mathrm{CXO}$ J1745-28 to the CWBs $\eta$ Carinae (e.g. Pittard \& Corcoran 2002) and Cyg OB2\#8A (DeBecker et al. 2006), we summarise the properties of WNLh CWBs in the Galaxy and Large Magellanic Cloud in Table 4. While the X-ray luminosity of the sample is observed to range over more than two orders of magnitude, WR25, BAT99-112 \& 116 are directly comparable to

$24 \mathrm{U} 1700-37-\mathrm{O}^{2} .5 \mathrm{Iaf}^{+}, P_{\text {orb }}=3.412 \mathrm{~d}$ (Clark et al. 2002); OAO1657-415 - WN9-11h, $P_{\text {orb }}=10.4$ d (Mason et al. 2009); GX301$2-$ B hypergiant, $P_{\text {orb }}=41.5 \mathrm{~d}$ (Kaper et al. 2006).

3 The accretors in GX301-2 and OAO1657-415 are pulsars, while the nature of 4U1700-37 is uncertain; however for the persistent Black Hole systems such as Cyg X-1 a composite multitemperature disc+power law spectrum is typically observed (e.g. Cui et al. 2002), which also appears to differ from CXO J1745-28. 
Table 4. Summary of the stellar, orbital and, where determined, the X-ray properties of confirmed or candidate massive WNLh binary systems in the Galaxy and LMC.

\begin{tabular}{|c|c|c|c|c|c|c|c|c|}
\hline$\overline{\mathrm{ID}}$ & $\begin{array}{l}\text { Spec. } \\
\text { type }\end{array}$ & $\begin{array}{c}\log \left(L_{W R}\right) \\
\left(L_{\odot}\right)\end{array}$ & $\begin{array}{c}M_{W R \text {,dynamical }} \\
\left(M_{\odot}\right)\end{array}$ & $\begin{array}{c}P_{\text {orb }} \\
\text { (days) }\end{array}$ & $\begin{array}{c}L_{\mathrm{x}} \\
\left(10^{33} \mathrm{erg} \mathrm{s}^{-1}\right)\end{array}$ & $\begin{array}{c}k T \\
(\mathrm{keV})\end{array}$ & $\begin{array}{c}\text { Fe sc XXV } \\
(\mathrm{Y} / \mathrm{N})\end{array}$ & References \\
\hline CXO J1745-28 & $\begin{array}{l}\text { WN9h + } \\
\text { mid-O? }\end{array}$ & $\begin{array}{c}6.24 \\
- \\
-\end{array}$ & $\begin{array}{l}- \\
- \\
-\end{array}$ & $189 \pm 6$ & 110 & $0.7+4.7$ & $\mathrm{Y}$ & 1,2 \\
\hline BAT99-116 (Mk34) & WN5h & - & - & $\mathrm{RV}$ var. & 240 & 3.9 & - & 3,4 \\
\hline WR25 (HD 93162) & WN6h + ? & & - & $207.8 \pm 0.3$ & $130 \pm 10$ & $0.7+2.8$ & $\mathrm{Y}$ & 5,6 \\
\hline BAT99-112 (R136c) & WN5h & - & - & RV var. & 110 & 3.0 & - & 3,7 \\
\hline NGC3603 A1 & $\begin{array}{l}\text { WN6ha + } \\
\text { WN6ha }\end{array}$ & $\begin{array}{c}6.2 \\
-\end{array}$ & $\begin{array}{c}116 \pm 31 \\
89 \pm 16\end{array}$ & $3.77 \pm ?$ & $>20$ & - & - & $8,9,10,11$ \\
\hline BАT99-99 (Mk39) & O3If*/WN6 & - & - & $92.6 \pm 0.3$ & $13 \pm 2$ & 1.6 & - & 4 \\
\hline WR20a & $\begin{array}{l}\text { WN6ha + } \\
\text { WN6ha }\end{array}$ & $\begin{array}{l}6.1 \\
6.1\end{array}$ & $\begin{array}{l}82.7 \pm 5.5 \\
81.9 \pm 5.5\end{array}$ & 3.7 & 8.0 & $0.35+1.55$ & - & $12,13,14$ \\
\hline BAT99-118 (R144) & WN6h & - & - & RV var. & 3.3 & 2.1 & - & 3,4 \\
\hline WR21a & $\begin{array}{c}\text { WN6ha }+ \\
\text { O4 }\end{array}$ & - & $\begin{array}{l}87 \pm 6 \\
53 \pm 4\end{array}$ & 31.7 & $0.4-1.6$ & 3.3 & - & 15,16 \\
\hline BAT99-119 (R145) & WN6h & - & - & 158.8 & 1.0 & 1.6 & - & 3,17 \\
\hline WR22 (HD 92740) & WN7h + & & $55.3 \pm 7.3$ & 80.3 & 0.9 & - & - & 18,19 \\
\hline BAT99-103 (R140b) & WN6h & - & - & 2.76 & $0.8 \pm 0.3$ & - & - & 4 \\
\hline WR 148 (HD 197406) & WN8h + ? & - & - & 4.32 & $0.6 \pm 0.3$ & - & - & $20,21,22$ \\
\hline BAT99-77 & WN7ha & - & - & 3.00 & 0.5 & - & - & 4 \\
\hline WR12 & WN8h + ? & - & - & 23.9 & 0.3 & - & - & $20,23,24$ \\
\hline BAT99-12 & O3If*/WN6 & - & - & 3.23 & $<6.0$ & - & - & 4 \\
\hline ВАТ99-32 & WN6(h) & - & - & 1.91 & $<4.5$ & - & - & 4 \\
\hline BAT99-113 (Mk30) & O3If*/WN6 & - & - & 4.70 & $<1.3$ & - & - & 4 \\
\hline BAT99-95 (R135) & WN7h & - & - & 2.11 & $<0.6$ & - & - & 4 \\
\hline Arches-F6 & WN9h & 6.3 & - & - & 11.0 & 1.9 & $\mathrm{Y}$ & 25,26 \\
\hline R136a3(bl) & WN5h & - & - & - & 8.5 & 4.2 & - & 3,7 \\
\hline WR20b & WN6ha & 5.9 & - & - & 6.5 & $0.5+5.5$ & - & 12,13 \\
\hline Arches-F7 & WN9h & 6.25 & - & - & 7.2 & 2.1 & $\mathrm{Y}$ & 25,26 \\
\hline Arches-F9 & WN9h & 6.3 & - & - & 4.6 & 3.3 & $\mathrm{Y}$ & 25,26 \\
\hline
\end{tabular}

Top panel: summary of the properties of CXO J1745-28 and X-ray bright $\left(>10^{35} \mathrm{erg} \mathrm{s}^{-1}\right)$ binary systems. Middle panel: remaining spectroscopically or photometrically confirmed binaries. Bottom panel: binary candidates selected on the basis of their X-ray properties (see Sect. 4). Stars are ordered in terms of decreasing X-ray flux, with errors given for this parameter and orbital period if provided in the original study. We quote dynamical masses only, with those given in italics representing lower limits. Regarding individual sources, WR20a, 22 and 25 have all been flagged as X-ray variable (Naze et al. 2008a; Gosset et al. 2003; Pollock \& Corcoran 2006), while the X-ray fluxes given for the Arches sources assume a 1T fit; a similar fit for CXO J1745-28 results in a directly comparable flux (Wang et al. 2006; Mikles et al. 2006). The converse is also true; the $2 \mathrm{~T}(0.9+5.8 \mathrm{keV})$ fit for F9 favoured by Yusef-Zadeh et al. (2002) yields $L_{\mathrm{x}} \sim 8 \times 10^{34} \mathrm{erg} \mathrm{s}^{-1}$; we choose to present the 1T fits for all three stars since Yusef-Zadeh et al. (2002) only present a spectral fit for F9. References for the values quoted in the table are: (1) Mikles et al. (2006); (2) Mikles et al. (2008); (3) Townsley et al. (2006); (4) Schnurr et al. (2008a); (5) Gamen et al. (2006); (6) Raassen et al. (2003); (7) Schnurr et al. (2009b); (8) Crowther \& Dessart (1998); (9) Schnurr et al. (2008b); (10) Moffat et al. (2002); (11) Moffat et al. (2004); (12) Naze et al. (2008a); (13) Tsujimoto et al. (2007); (14) Rauw et al. (2005); (15) Benaglia et al. (2005); (16) Niemela et al. (2008); (17) Schnurr et al. (2009a); (18) Schweickhardt et al. (1999); (19) Pollock (1987); (20) Hamann et al. (2006); (21) Pollock et al. (1995); (22) Drissen et al. (1986); (23) Lamontagne et al. (1996); (24) Ignace et al. (2000); (25) Martins et al. (2008) and (26) Wang et al. (2006).

CXO J1745-28. Likewise, where it may be determined, the emission from WNLh CWBs is significantly harder than $k T \sim$ $0.6 \mathrm{keV}$ expected for shock emission in a single stellar wind (e.g. Oskinova 2005), and for both WR20a and WR25 a two component fit is required, as found for CXO J1745-28. In addition to the comparable X-ray luminosity and spectral components, WR25 is of particular interest given the presence of strong Fe XXV emission, variable X-ray flux (Pollock \& Corcoran 2006) and a spectroscopically determined orbital period of $207.8 \pm 0.3 \mathrm{~d}$ (Raassen et al. 2003).

We consider these striking similarities as strong evidence for a CWB interpretation for CXO J1745-28. However, one would like to identify further examples of such systems in order to cement such an interpretation. Intriguingly, 3 of the 4 WN89h stars within the Arches (Table 4) which have X-ray detections also demonstrate similarly strong Fe XXV emission to CXO $\mathrm{J} 1745-28$ (the fourth, F2, being too faint to determine if this is the case). Spectral fits to these stars require a hard component for either a one (Wang et al. 2006) or two (Yusef-Zadeh et al. 2002) temperature model, with the resultant luminosities $-10^{33} \mathrm{erg} \mathrm{s}^{-1}$ and $10^{35} \mathrm{erg} \mathrm{s}^{-1}$ respectively - also directly comparable to that of CXO J1745-28 under the same model assumptions. Given the similarity of the stellar+wind properties of the Arches members to CXO J1745-28 (Sect 2.4 and Figs. 2, 4 and 5) we therefore conclude that these are physically identical systems. This in turn provides additional support for a CWB interpretation. The WN7-9ha stars within the Arches are the most massive stars present, and are at an age when it is not expected that any stars will have been lost to $\mathrm{SNe}$ (Table 5). Consequently, it is not apparent that an HMXB interpretation is possible on evolutionary grounds, still less since one would also have to conclude that the post SNe orbital configurations for CXO J1745-28 and Arches F6, $7 \& 9$ would have to be $\sim$ identical in order to reproduce their common X-ray properties.

Under the hypothesis that CXO J1745-28 is a CWB can we draw any conclusions as to the nature of the hitherto unseen 
companion? Given the absence of a radial velocity (RV) curve tailored hydrodynamical simulations are clearly premature but it is possible to estimate bulk properties for the secondary. Initially, the strong shock jump conditions $\left(k T=(3 / 16) \bar{m} v^{2}\right.$, where $\bar{m}$ is the mean particle mass $-10^{-27} \mathrm{~kg}$ for solar abundances (Stevens et al. 1992) - and the other symbols have their usual meanings) gives $v \sim 2100 \mathrm{~km} \mathrm{~s}^{-1}$. This is consistent with the mean terminal velocity of mid-late O stars (Prinja et al. 1990), but not with the W7-9h stars within the Arches (Martins et al. 2008), implying that a system consisting of twin WNLh stars such as WR20a is not viable.

We may use the analytical expressions from Pittard \& Stevens (2002) to estimate the X-ray flux for a representative $\mathrm{WN} 9 \mathrm{~h}+$ mid $\mathrm{O}$ star binary to test the consistency of such an hypothesis. Assuming $M_{\mathrm{WR}} \sim 80 M_{\odot}$ (Sect. 2.4 and Fig. 4), $M_{\mathrm{O}} \sim 30 M_{\odot}$ and an $189 \mathrm{~d}$ period we derive a binary separation of $\sim 5 \times 10^{13} \mathrm{~cm}$. We first define the momentum ratio of the two winds $\eta=\dot{M}_{\mathrm{O}} v_{\mathrm{O}} / \dot{M}_{\mathrm{WR}} v_{\mathrm{WR}}$. Then the kinetic power processed for each star is given by $L=0.5 \dot{M} v^{2} \Xi$, where the fractional wind kinetic power $\Xi$ is a function of $\eta$. With the properties of the primary from Table $2, v_{\mathrm{O}} \sim 2100 \mathrm{~km} \mathrm{~s}^{-1}$ and $\dot{M}$ in the range $2-20 \times 10^{-7} M_{\odot} \mathrm{yr}^{-1}$ we find $\eta=0.01-0.1$ and corresponding values for $\Xi_{\mathrm{WR}}=0.004-0.033$ and $\Xi_{\mathrm{O}}=0.564-0.403$ (Pittard $\&$ Stevens 2002). Then we may estimate the intrinsic X-ray luminosity for each star as $L_{\mathrm{x}}=0.5 \dot{M} v^{2} \Xi / \chi$, where the cooling efficiency, $\chi$, takes the conversion efficiency of kinetic wind power into radiation into account, and is given by $\chi=v_{8}{ }^{4} d_{12} / \dot{M}_{-7}$ (where $v_{8}$ is wind velocity in units of $1000 \mathrm{~km} \mathrm{~s}^{-1}, d_{12}$ is the distance from the star of the contact discontinuity in units of $10^{12} \mathrm{~cm}$ and $\dot{M}_{-7}$ the mass loss rate in units of $10^{-7} M_{\odot} \mathrm{yr}^{-1}$ ). Note that if $\chi<1.0$ it is set to 1.0 for the calculation of X-ray luminosity since one may not radiate more energy than one has as input (Pittard, priv. comm., 2009).

We estimate $d_{12}$ from the results presented in Pittard \& Stevens (2002) and then substituting the appropriate values into the above relationships we find $L_{X, W R} \sim 8 \times 10^{34} \mathrm{erg} \mathrm{s}^{-1}$ and $L_{\mathrm{X}, \mathrm{O}} \sim 9 \times 10^{34}$ and $3 \times 10^{33} \mathrm{erg} \mathrm{s}^{-1}$ for $\dot{M}=20-2 \times$ $10^{-7} M_{\odot} \mathrm{yr}^{-1}$. Therefore we consider the X-ray luminosity of CXO J1745-28 to be broadly consistent with that expected from a binary composed of a massive WN9h primary and a less evolved $\sim$ mid $\mathrm{O}$ star secondary in a $189 \mathrm{~d}$ period orbit, although a full hydrodynamical simulation would be required to extract accurate predictions for the properties of the secondary (cf. Pittard \& Corcoran 2002).

\section{Discussion and concluding remarks}

We present a tailored non-LTE analysis of the IR counterpart to the bright X-ray source CXO J1745-28, finding it to be a highly luminous, massive WN8-9h star, with physical parameters comparable to such stars in the Arches cluster. Furthermore, 3 of the 4 Arches members with X-ray detections - F6, 7 \& $9-$ also share remarkably similar X-ray fluxes and spectra to CXO J1745-28. While the near-IR spectra of these objects are consistent with originating in single stars, their X-ray properties clearly argue for binarity, with a comparison to known HMXBs and CWBs favouring the latter interpretation - indeed the Galactic WN6ha+? CWB system WR25 is a near twin of CXO J174528. Moreover, it appears difficult to reconcile both the youth and extreme mass inferred for CXO J1745-28 and the Arches sources with the requirement for a SN to have occurred in order to yield a relativistic companion - however we note that if they are $\mathrm{HMXBs}$ then they will provide a unique insight into the final
Table 5. Summary of confirmed and candidate WNLh binaries in young open clusters.

\begin{tabular}{cccccc}
\hline \hline Cluster & Age & Total & RV & \multicolumn{2}{c}{ Binaries } \\
& $(\mathrm{Myr})$ & & survey & Confirmed & Candidates \\
\hline NGC3603 & $1.3 \pm 0.3$ & 3 & 3 & 2 & 0 \\
Carina & $1.5 \pm 0.5$ & 3 & 3 & 2 & 0 \\
HM-1 & $2.2 \pm 0.5$ & 2 & 0 & - & 0 \\
NGC6231 & $2.7 \pm 0.5$ & 1 & 0 & - & 0 \\
Arches & $2.5 \pm 0.5$ & 13 & 0 & - & 3 \\
Wd 2 & $2.6 \pm 0.2$ & 2 & 1 & 1 & 1 \\
Bochum 7 & $2.8 \pm 0.5$ & 1 & 1 & 1 & 0 \\
R136/30 Dor & $>2$ & 23 & 23 & 8 & 1 \\
\hline Total & & 48 & 31 & 14 & 5
\end{tabular}

The cluster list follows that of Crowther et al. (2006), supplemented with the WNLh rich Arches (Figer et al. 2002) and 30 Dor (Walborn $\&$ Blades 1989) clusters. Column 4 summarises the number of stars in each cluster for which a RV survey has been carried out. Confirmed binaries were identified via photometric or spectroscopic evidence, while candidate binaries were selected if $L_{\mathrm{x}} \geq 10^{34} \mathrm{erg} \mathrm{s}^{-1}$ and/or $k T>4 \mathrm{keV}$, noting that such strict criteria would exclude known spectroscopic binaries such as WR20a and WR22 (Table 4). Given the different sensitivities of current RV and X-ray surveys (e.g. Pollock 1987; Pollock et al. 1995; Ignace et al. 2000; Oskinova 2005) we present results for individual clusters.

stages of stellar evolution for the most massive stars that appear able to form in the local Universe.

Nevertheless, under either HMXB or CWB hypothesis these four systems add to the growing population of binary WNLh stars in the galaxy and LMC, which are summarised in Table 4 and currently consist of an additional 19 confirmed and 2 candidates. With periods ranging from $\sim 1.9-208 \mathrm{~d}$, the most compact binaries may be candidates for the Case $\mathrm{M}$ evolution described by De Mink et al. (2009; in which tidal forces spin up the stars leading to significant rotational mixing), while the long period systems have yet to encounter mass transfer, thus having evolved as single stars, but ones for which dynamical mass estimates may be obtained.

In Table 5 we summarise the population of WNLh binaries in young massive clusters. This restricted population, rather than the complete census, was chosen since it excludes candidates, such as CXO J1745-28, which have been identified as WNLh stars because of their binary derived observational properties and hence would introduce a selection bias. Thus, of the $31 \mathrm{WNLh}$ stars for which a long term survey for RV variability has been performed, 14 have been identified as binaries resulting in a binary fraction of $\sim 45 \%$, which we consider a lower limit given the lack of sensitivity of current RV surveys to long period systems (e.g. $>200 \mathrm{~d} \&>40 \mathrm{~d}$ for 30 Dor and R136 proper; Schnurr et al. 2008a, 2009b) and the additional X-ray selected candidates.

This is consistent with other surveys which find similarly high percentages for OB and Wolf-Rayet stars, albeit for different samples of stars comprising either lower (Clark et al. 2008; Ritchie et al. 2009) or a wider range of masses (Kobulnicky \& Fryer 2007; Sana et al. 2008; Bosch et al. 2009). Such a binary fraction potentially presents important constraints on the formation mechanisms for very massive stars such as CXO J1745-28 and the Arches population. Not only does (accretion driven) radiation pressure have to be overcome to yield $\geq 80 M_{\odot}$ stars but within a short period of time either the formative processes or another mechanism - such as dynamical interactions during cluster core collapse (S. Goodwin, priv. comm., 2009) - yields significant numbers of very massive short period systems. 
Finally, referencing the issues raised in the introduction, the extreme mass inferred for CXO J1745-28, as well as the discovery of similar stars such as WR102ka (Barniske et al. 2008) reinforces the supposition of e.g. Mauerhan et al. (2007) that a diffuse, apparently isolated population of massive stars is found within the central $\sim 50 \mathrm{pc}$ of the Galactic centre in addition to the well know massive clusters. The origin of such stars is currently unclear; at $\sim 17 \mathrm{pc}$ the projected distance of CXO J1745-28 from the Arches - the only known GC cluster young enough for it to have formed in - appears uncomfortably large to explain its location as a result of dynamical ejection (implying a minimum travel time of $1.7 \mathrm{Myr}$ for a velocity of $10 \mathrm{~km} \mathrm{~s}^{-1}$ ). Finally, as with other massive star forming regions such as 30 Dor (Townsley et al. 2006), Wd 1 (Clark et al. 2008) and the putative complex at the base of the Scutum-Crux Arm (Clark et al. 2009) there are currently no unambiguous HMXB candidates within the Galactic Centre. Despite the high binary fractions inferred for massive stars within these regions, the physical processes leading to the production of neutron stars and black holes (binary mass transfer/common envelope evolution and supernovae) appear inimical to the production and/or retention of $\mathrm{X}$-ray bright HMXBs within their natal clusters/complexes.

Acknowledgements. J.S.C. acknowledges support from an RCUK fellowship, and thanks Julian Pittard, Ollie Schnurr, Selma de Mink, and Fabrice Martins for their invaluable help during the preparation of this manuscript and John Hillier for the use of his code.

\section{References}

Asplund, M., Grevesse, N., Sauval, A. J., Allende Prieto, C., \& Kiselman, D. 2004, A\&A, 417, 751

Audley, M. D., Nagase, F., Mitsuda, K., Angelini, L., \& Kelley, R. L. 2006, MNRAS, 367, 1147

Babel, J., \& Montmerle, T. 1997a, ApJ, 485, L29

Babel, J., \& Montmerle, T. 1997b, A\&A, 323, 121

Barniske, A., Oskinova, L. M., \& Hamann, W.-R. 2008, A\&A, 486, 971

Benaglia, P., Romeroa, G. E., Koribalski, B., \& Pollock, A. M. T. 2005, A\&A, 440,743

Bohannan, B., \& Crowther, P. A. 1999, ApJ, 511, 374

Bosch, G., Terlevich, R., Melnick, J., \& Selman, F. 1999, A\&AS, 137, 21

Bosch, G., Terlevich, E., \& Terlevich, R. 2009, AJ, 137, 3437

Bouret, J.-C., Donati, J.-F., Martins, F., et al. 2008, MNRAS, 389, 75

Casali, M., Adamson, A., \& Alves de Oliviera, C. 2007, A\&A, 467, 777

Clark, J. S., Goodwin, S. P., Crowther, P. A., et al. 2002, A\&A, 392, 909

Clark, J. S., Muno, M. P., Negueruela, I., et al. 2008, A\&A, 477, 147

Clark, J. S., Negueruela, I., \& Davies, B. 2009, A\&A, 498, 109

Cotera, A. S., Erickson, E. F., Colgan, S. W. J., et al. 1996, ApJ, 461, 750

Cox, A. 2000, Allen's Astrophysical Quantities

Crowther, P. A., \& Dessart, L. 1998, MNRAS, 296, 622

Crowther, P. A., Hadfield, L. J., Clark, J. S., Negueruela, I., \& Vacca, W., D. 2006, MNRAS, 372, 1407

Cui, W., Feng, Y. X., Zhang, S. N., et al. 2002, ApJ, 576, 357

DeBecker, M., Rauw, G., Sana, H., et al. 2006, MNRAS, 371, 1280

De Mink, S. E., Cantiello, M., Langer, N., et al. 2009, A\&A, 497, 243

Donati, J.-F., Babel, J., Harries, T. J., et al. 2002, MNRAS, 333, 55

Donati, J.-F., Howarth, I. D., Bouret, J.-C., et al. 2006, MNRAS, 365, L6

Drissen, L., Lamontagne, R., Moffat, A. F. J., Bastien, P., \& Seguin, M., 1986, ApJ, 304, 188

Figer, D. F., Morris, M., \& McLean, I. S. 1996, ASPC, 102, 263

Figer, D. F., Najarro, F., \& Gilmore, D. 2002, ApJ, 581, 258

Gagné, M., Oksala, M. E., Cohen, D. H., et al. 2005, ApJ, 628, 986

Gamen, R., Gosset, E., Morrell, N., et al. 2006, A\&A, 460, 777

Gosset, E., Rauw, G., Vreux, J.-M., et al. 2003, IUAS, 212, 188

Hamann, W. R., Grafener, G., \& Liermann, A. 2006, A\&A, 457, 1015

Hewett, P. C., Warren, S. J., Leggett, S. K., \& Hodgkin, S. T. 2006, MNRAS, 367,454

Hillier, D. J., \& Miller, D. L. 1998, ApJ, 496, 407

Hillier, D. J., \& Miller, D. L. 1999, ApJ, 519, 354

Hillier, D. J., Lanz, T., Heap, S. R., et al. 2003, ApJ, 588, 1039

Hodgkin, S. T., Irwin, M. J., Hewett, P. C., \& Warren, S. J. 2009, MNRAS, 394, 675

Hubrig, S., Scholler, M., Schnerr, R. S., et al. 2008, A\&A, 490, 793
Ignace, R., Oskinova, L. M., \& Foullon, C. 2000, MNRAS, 318, 214 Indebetouw, R., Mathis, J. S., \& Babler, B. L. 2005, ApJ, 619, 931 Kaper, L., van der Meer, A., \& Najarro, F. 2006, A\&A, 457, 505

Kim, S. S., Figer, D. F., Kudritzki, R. P., \& Najarro, F. 2006, ApJ, 653, L113

Kobulnicky, H. A., \& Fryer, C. L. 2007, ApJ, 670, 747

Krabbe, A., Genzel, R., Eckart, A., et al. 1995, APJ, 447, L95

La Barbera, A., Segreto, A., Santangelo, A., Kreykenbohm I., \& Orlandini, M. 2005, A\&A, 438, 617

Lamontagne, R., Moffat, A. F. J., Drissen, L., et al. 1996, AJ, 112, 2227

Lanz, T., \& Hubeny, I. 2003, ApJS, 146, 417

Lawrence, A., Warren, S. J., Almaini, O., et al. 2007, MNRAS, 379, 1599

Martins, F., \& Plez, B. 2006, A\&A, 457, 637

Martins, F., Schaerer, D., \& Hillier, D. J. 2005, A\&A, 441, 735

Martins, F., Hillier, D. J., \& Paumard, T. 2008, A\&A, 478, 219

Mason, A. B., Clark, J. S., Norton, A. J., Negueruela, I., \& Roche, P. 2009, A\&A, 505,281

Mauerhan, J. C., Muno, M. P., \& Morris, M. 2007, ApJ, 662, 574

Meynet, G., \& Maeder, A. 2000, A\&A, 361, 101

Mikles, V. J., Eikenberry, S. S., Muno, M. P., Bandyopadhyay, R. M., \& Patel, S. 2006, ApJ, 651, 408

Mikles, V. J., Eikenberry, S. S., Bandyopadhyay, R. M., \& Muno, M. P. 2008, ApJ, 689, 1222

Moffat, A. F. J. 2008, RMxAC, 33, 95

Moffat, A. F. J., Corcoran, M. F., Stevens, I. R., et al. 2002, ApJ, 573, 191

Moffat, A. F. J., Poitras, V., Marchenko, S. V., et al. 2004, AJ, 128, 2854

Montes, G., Perez-Torres, M. A., Alberdi, A., \& Gonzalez, R. F. 2009, ApJ, 705, 899

Morris, P. W., van der Hucht, K. A., \& Crowther, P. A. 2000, A\&A, 353, 624

Muno, M. P., Bauer, F. E., Bandyopadhyay, R. M., \& Wang, Q. D. 2006a, ApJS, 165,173

Muno, M. P., Bower, G. C., Burgasser, A. J., et al. 2006b, ApJ, 638, 183

Muno, M. P., Bauer, F. E., \& Baganoff, F. K. 2009, ApJS, 181, 110

Nagata, T., Woodward, C. E., Shure, M., \& Kobayashi, N. 1995, AJ, 109, 1676

Náze, Y., Rauw, G., Vreux, J.-M., \& DeBecker, M. 2004, A\&A, 417, 667

Náze, Y., Rauw, G., Pollock, A. M. T., Walborn N. R., \& Howarth, I. D. 2007, MNRAS, 375, 145

Náze, Y., Rauw, G., \& Manfroid, J. 2008b, A\&A, 483, 171

Náze, Y., Walborn, N. R., \& Martins, F. 2008b, RMxAA, 44, 331

Náze, Y., Walborn, N. R., Rauw, G., et al. 2008c, AJ, 135, 1946

Niemela, V. S., Gamen, R. C., Barba, R. H., et al. 2008, MNRAS, 389, 1447

Oskinova, L. M. 2005, MNRAS, 361, 679

Pittard, J. M., \& Corcoran, M. F. 2002, A\&A, 383, 636

Pittard, J. M., \& Stevens. I. R. 2002, A\&A, 388, L20

Pollock, A. M. T., ApJ, 320, 283

Pollock, A. M. T., \& Corcoran, M. F. 2006, A\&A, 1093

Pollock, A. M. T., Haberl, F., \& Corcoran, M. F. 1995, IAUS, 163, 522

Prinja, R. K., Barlow, M. J., \& Howarth, I. D. 1990, ApJ, 361, 607

Portegies Zwart, S. F., Pooley, D., \& Lewin, W. H. G. 2002, ApL, 574, 762

Raassen, A. J. J., van der Hucht, K. A., \& Mewe, R. 2003, A\&A, 402, 653

Raassen, A. J. J., van der Hucht, K. A., Miller, N. A., \& Cassinelli, J. P. 2008, A\&A, 478, 513

Rauw, G., Vreux, J.-M., Gosset, E., et al. 1996, A\&A, 306, 771

Rauw, G., Blomme, R., Walborn, W. L., et al. 2002, A\&A, 394, 993

Rauw, G., Crowther, P. A., De Becker, M., et al. 2005, A\&A, 432, 985

Rayner, J. T., Toomey, D. W., Onaka, P. M., et al. 2003, PASP, 115, 362

Reid, M. J. 1993, ARA\&A, 31, 345

Ritchie, B. W., Clark, J. S., Negueruela, I., \& Crowther, P. A. 2009, A\&A, 507, 1585

Sana, H., Gosset, E., Naze, Y., Rauw, G, \& Linder, N. 2008, MNRAS, 386, 447

Schnurr, O., Moffat, A. F. J., St-Louis, N., Morrell, N. I., \& Guerrero, M. A. 2008a, MNRAS, 389, 806

Schnurr, O., Casoli, J., Chene, A.-N., Moffat, A. F. J., \& St-Louis, N. 2008b, MNRAS, 389, L38

Schnurr, O., Moffat, A. F. J., Villar-Sbaffi, A., St-Louis, N., \& Morrell, N. I. 2009a, MNRAS, 395, 823

Schnurr, O., Chene, A.-N., Casoli, J., Moffat, A. F. J., \& St-Louis, N. 2009b, MNRAS, submitted

Schweickhardt, J., Schmutz, W., Stahl, O., Szeifert, Th, \& Wolf, B. 1999, A\&A, 347,127

Stevens, I. R., Blondon, J. M., \& Pollock, A. M. T. 1992, ApJ, 386, 265

Townsley, L. K., Broos, P. S., Feigelson, E. D., Garmire, G. P., \& Getman, K. V. 2006, AJ, 131, 2164

Tsujimoto, M., Feigelson, E. D., \& Townsley, L. K. 2007, ApJ, 665, 719

Yusef-Zadeh, F., Law C., Wardle, M., et al. 2002, ApJ, 570, 665

Walborn, N. R. 1982, ApJ, 256, 452

Walborn, N. R., \& Blades, J. C. 1997, ApJS, 112, 457

Wang, Q. D., Dong, H., \& Lang, C. 2006, MNRAS, 371, 38

White, N. E., Kallman, T. R., \& Swank, J. H. 1983, ApJ, 269, 264 